\title{
Clinical Relevance of Anti-TNF Antibody Trough Levels and Anti-Drug Antibodies in Treating Inflammatory Bowel Disease Patients
}

\author{
Ilana Reinhold ${ }^{a, b}$ Sena Blümel ${ }^{a}$ Jens Schreiner ${ }^{c}$ Onur Boyman ${ }^{c, d}$ \\ Jan Bögeholz ${ }^{a, b}$ Marcus Cheetham ${ }^{b}$ Gerhard Rogler ${ }^{a, d}$ Luc Biedermann ${ }^{a}$ \\ Michael Scharl ${ }^{\mathrm{a}}$ \\ aDepartment of Gastroenterology and Hepatology, University Hospital Zurich, University of Zurich, \\ Zurich, Switzerland; 'bepartment of Internal Medicine, University Hospital Zurich, University of Zurich, \\ Zurich, Switzerland; 'Department of Immunology, University Hospital Zurich, Zurich, Switzerland; \\ ${ }^{d}$ Faculty of Medicine, University of Zurich, Zurich, Switzerland
}

\section{Keywords}

Inflammatory bowel disease - Trough level measurement · Anti-drug antibodies · Crohn's disease - Ulcerative colitis · Anti-tumor necrosis factor · Infliximab · Adalimumab

\begin{abstract}
Background and Aims: The majority of patients treated with anti-tumor necrosis factor (TNF) therapy develop anti-drug antibodies (ADAs), which might result in loss of treatment efficacy. Strict guidelines on measuring trough levels (TLs) and ADA in clinical routine do not exist. To provide realworld data, we took advantage of our tertiary inflammatory bowel disease (IBD) center patient cohort and determined indicators for therapeutic drug monitoring (TDM) and actual consequences in patient care. Methods: We retrospectively collected clinical data of 104 IBD patients treated with infliximab or adalimumab in our IBD clinic. Patients with TL and ADA measurements between June 2015 and February 2018 were included. Results: The main reason for determining TL was increased clinical disease. Subtherapeutic TLs were found in 33 patients, therapeutic TLs in 33 patients, and supratherapeutic TLs in 38 patients. Adjustments in anti-TNF therapy occurred more frequently $(p=0.01)$ in patients with
\end{abstract}

karger@karger.com www.karger.com/iid

Karger $\frac{1}{\%}$

GOPEN ACCESS
(C) 2020 The Author(s)

Published by S. Karger AG, Basel

This is an Open Access article licensed under the Creative Commons Attribution-NonCommercial-4.0 International License (CC BY-NC) (http://www.karger.com/Services/OpenAccessLicense), applicable to the online version of the article only. Usage and distribution for commercial purposes requires written permission. subtherapeutic TL (24 of 33 patients; $73 \%$ ) as compared to patients with therapeutic and supratherapeutic TLs ( 26 of 71 patients; 37\%). No correlation could be found between TL and disease activity $(p=0.16)$. Presence of ADA was found in 16 patients, correlated with the development of infusion reactions (OR: 10.6, RR: 5.4, Cl: 2.9-38.6), and was associated with subtherapeutic TL in 15 patients (93.8\%). Treatment adaptations were based on TL and/or ADA presence in 36 of 63 patients. Conclusions: TDM showed significant treatment adaptations in patients with subtherapeutic TL. Conversely, in patients with therapeutic and supratherapeutic TLs, reasons for adaptations were based on considerations other than $\mathrm{TL}$, such as clinical disease activity. Further studies should focus on decision-making in patients presenting with supratherapeutic TL in remission.

(c) 2020 The Author(s)

Published by S. Karger AG, Basel

\section{Introduction}

Anti-tumor necrosis factor (TNF) antibodies, such as infliximab and adalimumab, are important medications for the treatment of patients with inflammatory bowel disease (IBD). However, biological agents (biologicals) 
are costly, thus burdening the health care system [1]. The development of sustained anti-drug antibodies (ADAs) occurs in up to $73 \%$ of patients treated with infliximab and up to $35 \%$ of patients treated with adalimumab [2]. The presence of ADA commonly results in a loss of response, which usually occurs within 12 months after the onset of treatment in about one-third of patients [2]. Moreover, ADA can cause other adverse events, such as infusion reactions $[3,4]$. Therefore, the measurement of anti-TNF antibody trough levels (TL) and the determination of $\mathrm{ADA}$ are frequently performed to optimize the management of patients with IBD [5-7]. Therapeutic drug monitoring (TDM) can be done to either detect a possible reason for treatment failure or loss of response (so-called "reactive" TDM), or to optimize therapy outcome and prevent flares or loss of response (so-called "proactive" TDM) [2].

Although the European Crohn's and Colitis Organization (ECCO) states the existence of a positive doseresponse relationship between TL and clinical outcomes, the ECCO does not provide a detailed recommendation on when to measure TL or ADA in the clinical routine setting [8]. However, recent consensus panels proposed to determine TL and ADA after successful induction and when treatment failure occurs $[2,9]$. In addition, measurement of $\mathrm{ADA}$ is recommended before reinitiating anti-TNF therapy to avoid an acute infusion reaction [10]. The development of ADA can be reduced by concomitant treatment with immunomodulators (i.e., thiopurines or methotrexate [MTX]) [11]. Alternatively, ADA levels may increase or even disappear spontaneously over time without any clinical relevance [12]. Regarding TL of infliximab or adalimumab, it is recommended to maintain high levels because these were found to be beneficial for endoscopic remission in luminal IBD $[2,11,13,14]$. However, no official recommendation exists about the optimal range of TL to maintain clinical remission. In addition, the consequences of TL measurements, including dosing or time interval adaptation of the biological, change of the biological, and addition or alterations of immunomodulatory treatments, have only been described in a few studies [15-17].

Thus, due to a lack of common guidelines, it is unclear at present, in which clinical settings TL and ADA should be measured and which consecutive treatment adaptations are warranted. Therefore, we aimed to analyze the reasons for and consequences of TL and ADA measurements in the clinical real-life setting in our large tertiary Swiss IBD center at the University Hospital Zurich.

Trough Level and Anti-Drug Antibody Measurement in IBD

\section{Methods}

\section{Study Design}

We retrospectively analyzed the charts of 104 IBD patients who had measurements of TL and ADA when attending our tertiary care IBD center at the University Hospital Zurich (USZ), Switzerland, between June 2015 and February 2018. All enrolled patients were participants of the Swiss IBD Cohort study, which was approved by the Ethics Committee of the Canton Zurich (No. EK-1316). Every patient was treated with infliximab or adalimumab. Patient characteristics and additional IBD-related treatments were extracted from the electronic health record system of the USZ. Disease classification at the time of serum analysis was documented by the Montreal classification, including disease severity by either the Harvey-Bradshaw Index (HBI) for Crohn's disease (CD) or the Modified Truelove and Witts Severity Index score (MTWSI) for ulcerative colitis (UC) $[18,19]$. Only one measurement per patient was taken for analysis in this study, namely the first measurement that was performed within the time period of data analysis. Further measurements of TL in the patients were analyzed but not included in the initial analysis due to the diversity and disparity of patients' follow-up.

The detailed reasons for performing TL and ADA measurements were subdivided into 10 different groups based on the notes of the treating physician: (1) clinical flare, (2) sonographic activity, (3) endoscopic activity, (4) elevated laboratory parameters (C-reactive protein and fecal calprotectin), (5) TL monitoring without any ongoing adaptation of dosing (quiescent disease), (6) TL monitoring during ongoing adaptation of dosing, (7) TL monitoring at the end of treatment induction, (8) recent presence of ADA, (9) suspected side effect of anti-TNF therapy or suspected presence of ADA, and (10) verification of potential ADA development due to former exposure to the same biological prior to reinitiation. TL results for infliximab and adalimumab were classified into 3 subgroups specified as "subtherapeutic" (infliximab $<3.5 \mu \mathrm{g} / \mathrm{L}$ and adalimumab $<5 \mu \mathrm{g} / \mathrm{L}$ ), "therapeutic" (infliximab 3.5-7 $\mu \mathrm{g} / \mathrm{L}$ and adalimumab 5-8 $\mu \mathrm{g} / \mathrm{L}$ ), and "supratherapeutic" (infliximab $>7 \mu \mathrm{g} / \mathrm{L}$ and adalimumab $>8 \mu \mathrm{g} / \mathrm{L}$ ), based on the publication of Vande Casteele and colleagues [20].

The performed treatment adaptations following the results of TL and ADA measurements were subdivided into the following 3 groups: (1) adaptation of anti-TNF therapy (dose or interval change, stopping ongoing anti-TNF therapy, or change to another biological treatment, such as infliximab, adalimumab, golimumab, and vedolizumab), (2) treatment changes other than anti-TNF therapy (initiation of immunomodulators, e.g., azathioprine [AZA] or MTX; corticosteroids [topical or systemic]; surgery; or antibiotics), and (3) no treatment adaptation. One patient could belong to groups (1) and (2) if both anti-TNF therapy adaptation and addition of immunomodulators were applied.

Physicians' notes available from the electronic health record system were analyzed to define if performed treatment adaptations were based on the results from TL and ADA measurements or if these decisions were established based on clinical signs and other investigations, such as laboratory results or sonographic or endoscopic appearance, regardless of TL and ADA results. If follow-up measurements were performed, the interval between TL measurements, development of $\mathrm{ADA}$, and therapeutic consequences in case of ADA development were recorded. 
Table 1. Clinical and demographic patient characteristics at first trough level measurements

\begin{tabular}{|c|c|c|c|c|c|c|c|c|c|}
\hline & \multicolumn{4}{|c|}{$\mathrm{UC}(n=30)$} & \multicolumn{4}{|c|}{$\mathrm{CD}(n=74)$} & \multirow{2}{*}{$\begin{array}{l}p \\
\text { value }\end{array}$} \\
\hline & cou & $\%$ & mea & range & count & $\%$ & mean $\mathrm{r}$ & range & \\
\hline \multicolumn{10}{|l|}{ Sex } \\
\hline Female & 14 & 47 & & & 39 & 53 & & & \\
\hline Male & 16 & 57 & & & 35 & 47 & & & \\
\hline Age at measurement, years & & & 38 & $(17-74)$ & & & 35 & $(12-70)$ & 0.49 \\
\hline Age at diagnosis, years & & & 30 & $(12-68)$ & & & 25 & $(6-63)$ & 0.22 \\
\hline \multicolumn{10}{|l|}{ Smoker } \\
\hline No & 26 & 87 & & & 49 & 67 & & & 0.03 \\
\hline Yes & 4 & 13 & & & 24 & 33 & & & \\
\hline \multicolumn{10}{|c|}{ Extraintestinal manifestations (joint, skin, and ocular) } \\
\hline No & 15 & 50 & & & 45 & 62 & & & 0.19 \\
\hline Yes & 15 & 50 & & & 28 & 39 & & & \\
\hline \multicolumn{10}{|l|}{ Surgery } \\
\hline No & 27 & 90 & & & 32 & 44 & & & $<0.01$ \\
\hline Yes & 3 & 10 & & & 41 & 56 & & & \\
\hline \multicolumn{10}{|l|}{ Anti-TNF therapy } \\
\hline Infliximab & 22 & 73 & & & 42 & 57 & & & 0.09 \\
\hline Adalimumab & 8 & 27 & & & 32 & 43 & & & \\
\hline Infliximab dosage, $\mathrm{mg} / \mathrm{kg}$ & & & 5 & $(5-10)$ & & & 6 & $(5-10)$ & \\
\hline Infliximab treatment interval, days & & & 47 & $(28-56)$ & & & 49 & $(14-56)$ & \\
\hline Adalimumab dosage, $\mathrm{mg}$ & & & 40 & $(40)$ & & & 43 & $(40-80)$ & \\
\hline Adalimumab treatment interval, days & & & 12 & $(7-14)$ & & & 14 & $(7-21)$ & \\
\hline \multicolumn{10}{|c|}{ Immunomodulators or other concomitant treatments } \\
\hline None of the mentioned treatments & 1 & 3 & & & 41 & 55 & & & \\
\hline AZA & 3 & 10 & & & 7 & 10 & & & \\
\hline MPU & 1 & 3 & & & 1 & 1 & & & \\
\hline Corticosteroids, syst & 3 & 10 & & & 6 & 8 & & & \\
\hline Methotrexate & 1 & 3 & & & 2 & 3 & & & \\
\hline Corticosteroids, topical & 0 & & & & 3 & 4 & & & \\
\hline Antibiotics & 0 & & & & 3 & 4 & & & \\
\hline 5 -ASA & 8 & 27 & & & 2 & 3 & & & \\
\hline Corticosteroids syst and antibiotics & 0 & & & & 2 & 3 & & & \\
\hline Corticosteroids syst and AZA or MPU & 4 & 13 & & & 2 & 3 & & & \\
\hline Corticosteroids syst and MTX & 1 & 3 & & & 2 & 3 & & & \\
\hline Corticosteroids syst and 5-ASA & 6 & 20 & & & 1 & 1 & & & \\
\hline Others $^{1}$ & 2 & 7 & & & 2 & 3 & & & \\
\hline MTWSI at presentation & & & 7 & $(1-16)$ & & & & & \\
\hline \multicolumn{10}{|l|}{ Montreal classification UC (E1-E3) } \\
\hline E1: Proctitis & 4 & 13 & & & & & & & \\
\hline E2: Left-sided colitis & 13 & 43 & & & & & & & \\
\hline E3: Pancolitis & 13 & 43 & & & & & & & \\
\hline HBI at presentation & & & & & & & 6 & $(1-14)$ & \\
\hline \multicolumn{10}{|l|}{ Montreal classification CD: location (L1-L4) } \\
\hline L1 (ileal) & & & & & 14 & 19 & & & \\
\hline L2 (colonic) & & & & & 22 & 30 & & & \\
\hline L3 (ileocolonic) & & & & & 35 & 47 & & & \\
\hline L4 (isolated upper disease) & & & & & 3 & 4 & & & \\
\hline \multicolumn{10}{|c|}{ Montreal classification CD: behavior (B1-B3) } \\
\hline B1 (nonstricturing, nonpenetrating) & & & & & 27 & 36 & & & \\
\hline B2 (stricturing) & & & & & 7 & 9 & & & \\
\hline B3 (penetrating) & & & & & 40 & 54 & & & \\
\hline
\end{tabular}

$\mathrm{CD}$, Crohn's disease; UC, ulcerative colitis; TNF, tumor necrosis factor; AZA, azathioprine; MPU, mercaptopurine; syst, systemic; 5-ASA, 5-aminosalicylic acid; MTX, methotrexate; MTWSI, Modified Truelove and Witts Severity Index score; HBI, Harvey-Bradshaw Index. ${ }^{1}$ Others: tacrolimus, vedolizumab, study drug, or cyclosporine. 
Fig. 1. Reasons for TL measurements $(n=$ 104, CD and UC patients): clinical flare (a) (i.e., symptoms or signs for disease activity) was the main reason for measurement of TL, followed by TL monitoring without treatment adaptation (b) (quiescent disease and proactive monitoring). Suspected side effect of anti-TNF therapy or suspected presence of ADAs (c); elevated laboratory parameters (C-reactive protein and fecal calprotectin) (d); sonographic activity (e); endoscopic activity (f); TL monitoring at the end of treatment induction $(\mathbf{g})$; verification of potential ADA development due to former exposure to the same biological before reinitiation $(\mathbf{h})$; recent presence of ADA (i); TL monitoring with ongoing adaptation of dosing $(\mathbf{j})$. TL, trough level; CD, Crohn's disease; UC, ulcerative colitis; TNF, tumor necrosis factor; ADAs, anti-drug antibodies.

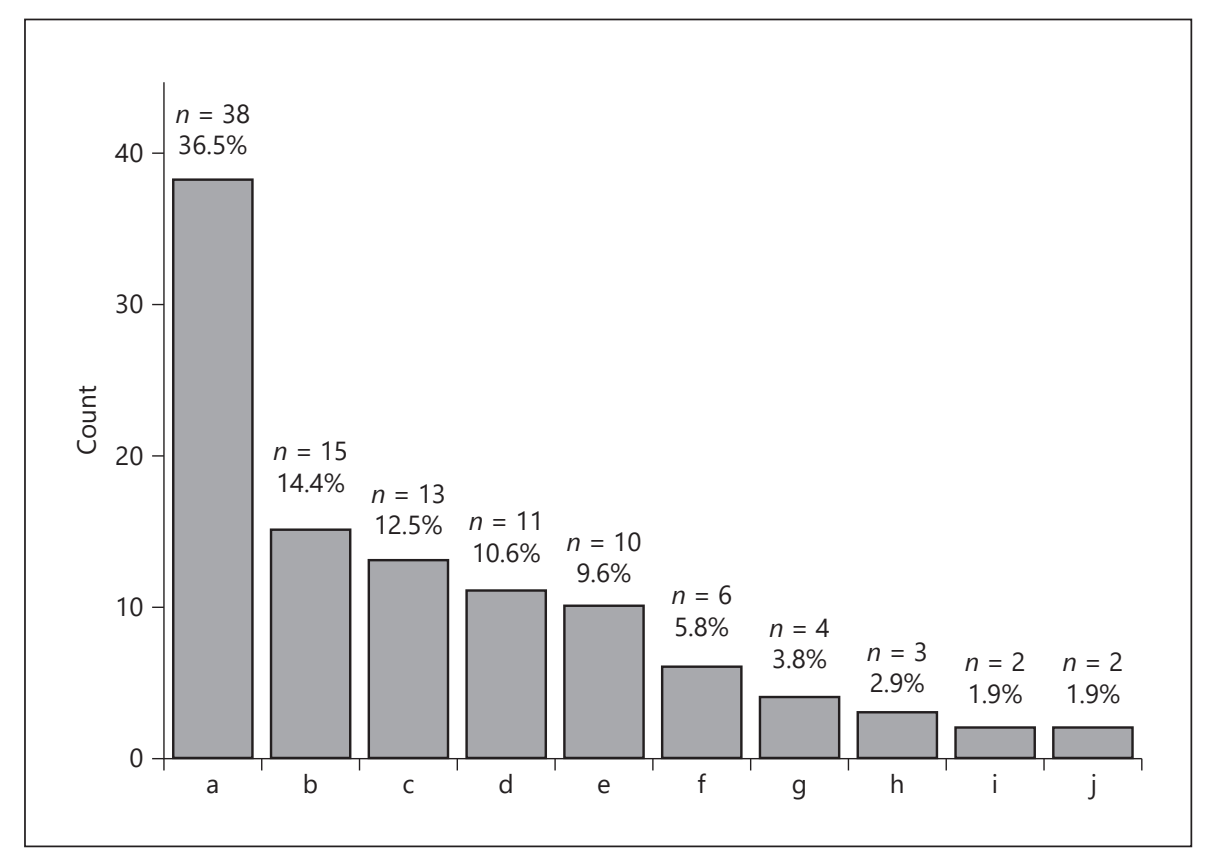

Detection of Infliximab and Adalimumab TLs and ADAs

Serum for TL and ADA analysis was obtained immediately before the next infliximab or adalimumab application or on the day of admission for hospitalized patients. TL and ADA levels for infliximab were measured using a commercially available ELISA kit (Lisa Tracker Duo Infliximab, Theradiag, France), which is a drug-sensitive assay. The normal range for infliximab TL was set to $3.5-7 \mu \mathrm{g} / \mathrm{L}$, consistent with a previous publication [14]. ADAs against infliximab were categorized into present or negative $(<10$ $\mathrm{ng} / \mathrm{mL}$ ). TL and ADA levels for adalimumab were analyzed using a commercially available ELISA kit (Lisa Tracker Duo Adalimumab, Theradiag, France), a drug-sensitive assay. The normal range for adalimumab TL was determined to be $5-8 \mu \mathrm{g} / \mathrm{L}$ [14]. ADAs against adalimumab were categorized into present or negative $(<10 \mathrm{ng} / \mathrm{mL})$. Due to similar assay characteristics, TL and ADA measurements were always performed in parallel. All analyses were routinely performed by the Department of Immunology at the University Hospital Zurich.

\section{Data Analysis and Statistics}

Data were analyzed using SPSS version 25.0 (IBM Corp., Armonk, NY, USA). $p<0.05$ was considered statistically significant. Patients' baseline characteristics in Table 1 were analyzed by Mann-Whitney $U$ test and $\chi^{2}$ test for continuous and categorical data, respectively.

For analysis of the performed treatment adaptations, we used $\chi^{2}$ test and the exact binomial test, including the analysis of the 3 possible TL results (sub-, supra-, or therapeutic), and subsequently performed changes in anti-TNF therapy: (1) yes, meaning adaptation performed by higher dosing, interval shortening or lengthening, stopping or switching of biological treatment; (2) no, meaning no treatment adaptation regarding anti-TNF therapy; analysis of correlation between clinical surrogate for increased disease activity (clinical flare, a sonographic or endo- scopic activity, or elevated laboratory parameters) and the 3 possible TL results; analysis regarding physicians' motivation for consequent treatment adaptations compared to the 3 TL results. Odds ratios were calculated for the presence of ADA and adverse drug reactions. Follow-up data were analyzed using a paired sample $t$ test.

\section{Results}

\section{Patient Characteristics}

Demographic details and patient characteristics are presented in Table 1. A total of 104 patients (CD: $n=74$; UC: $n=30$ ) were included. The majority was regularly seen in an ambulatory outpatient setting $(n=87)$ in the context of a specialized tertiary IBD clinic. A subset of patients $(n=17)$ was hospitalized due to disease flares needing intravenous antibiotics or corticosteroids. One of those patients was hospitalized in the intensive care unit due to a septic shock. Three patients received both infliximab and adalimumab in succession if one of the drugs lost effectiveness. The frequency of treatment with either infliximab or adalimumab in CD or UC was not different $(p=0.08)$. The dosage of infliximab ranged from 5 to $10 \mathrm{mg} / \mathrm{kg}$ body weight and was administered in intervals of 4-8 weeks. Adalimumab was mostly given by the standard maintenance dosage of $40 \mathrm{mg}$ every other week. 


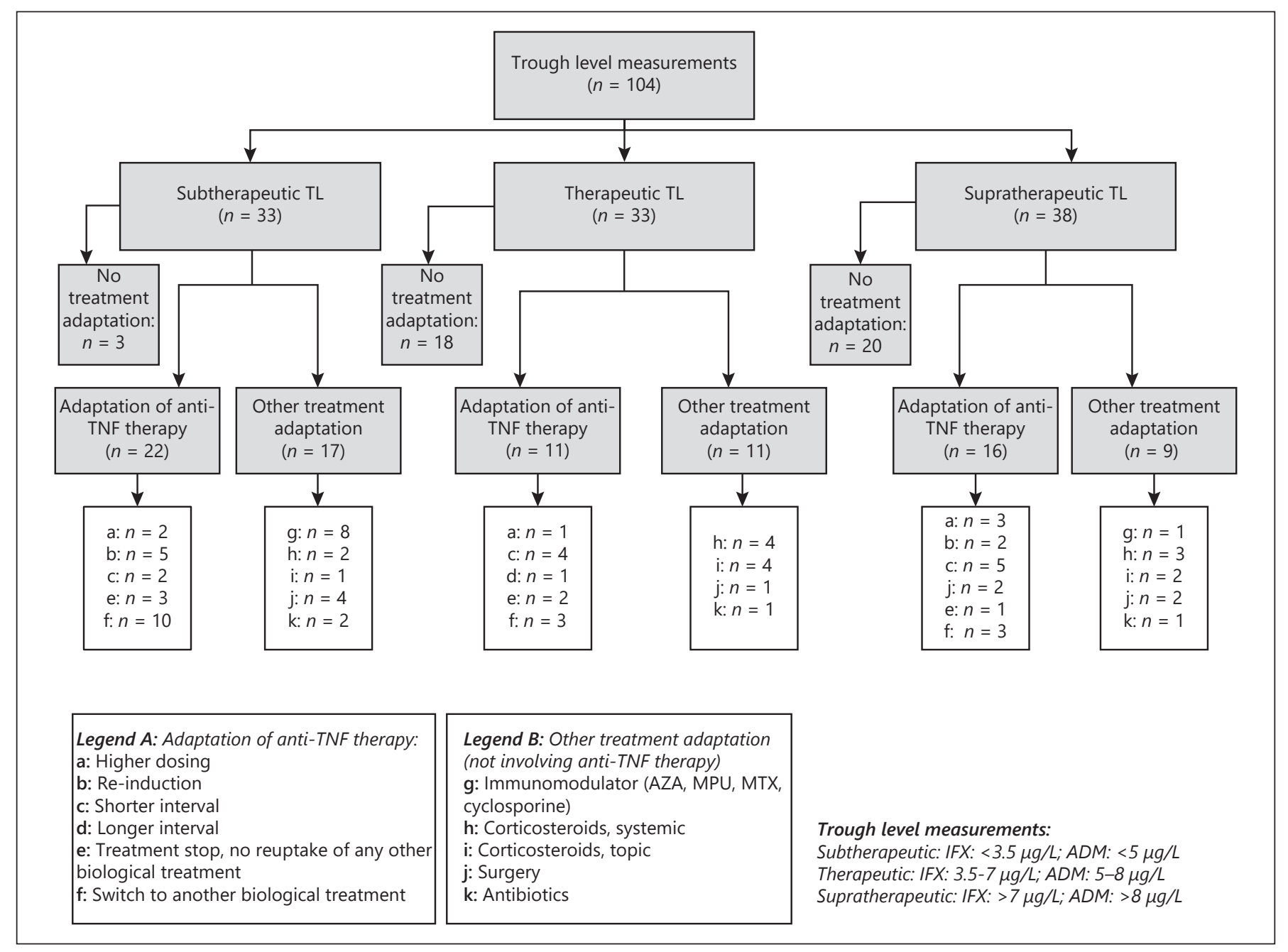

Fig. 2. Results of TL measurements and resulting therapeutic consequences: treatment adaptations (regarding anti-TNF therapy and other adaptations) were mainly performed in patients presenting with subtherapeutic TLs. One patient could have received both changes in anti-TNF therapy and other treatment adaptations. TL, trough level; TNF, tumor necrosis factor.

\section{Reasons for TL Measurements}

The detailed reasons for TL measurements are represented in Figure 1. TL measurements were mainly performed in patients presenting with a clinical flare ( $38 \mathrm{pa}-$ tients, $36.5 \%)$, such as higher frequency of diarrhea, bloody stools, abdominal cramps, or painful abdominal palpation indicative for increased disease activity. Interestingly, proactive TDM was the second leading reason for TL measurements (15 patients, 14.4\%), while other signs of enhanced disease activity, such as elevated CRP or calprotectin levels, sonographic or endoscopic disease activity, or suspected side effects, were less often the reason for TL determination.

\section{TL Results and Subsequent Treatment Adaptations}

An overview of TLs and their impact on treatment decisions are given in Figure 2. One patient could undergo both a change in anti-TNF therapy and other treatment adaptations, such as change or addition of immunomodulators and corticosteroids.

Subtherapeutic TLs were found in 33 patients. Out of the 22 patients with anti-TNF therapy changes, 9 (41\%) received a treatment intensification (i.e., higher dosing, reinduction of therapy, or interval shortening). Seventeen out of 33 patients (52\%) with subtherapeutic TL had (additionally) other treatment adaptations not involving anti-TNF therapy, such as introduction of immunomodulators or corticosteroids in the therapy. 
Table 2. Adaptation of anti-TNF therapy following TL measurements: treatment changes regarding anti-TNF therapy (i.e., alteration of treatment interval, dosing, stopping or switching to another biological therapy) were only significant in patients with subtherapeutic TL results

\begin{tabular}{lccc}
\hline & $\begin{array}{l}\text { Adaptation of } \\
\text { anti-TNF therapy }\end{array}$ & $p$ value \\
\cline { 2 - 3 } & no $(n) \quad$ yes $(n)$ & \\
\hline$T L$ & 9 & 24 & 0.01 \\
Subtherapeutic & 22 & 11 & 0.08 \\
Therapeutic & 23 & 15 & 0.26 \\
Supratherapeutic & 23 & \\
\hline
\end{tabular}

TNF, tumor necrosis factor; TL, trough level.

Therapeutic TLs were found in 33 patients and frequently resulted in no treatment adaptations at all. However, in patients presenting with a clinical flare, anti-TNF therapy was intensified together with initiation of topical $(n=4)$ or systemic $(n=4)$ corticosteroids.

Supratherapeutic TLs were found in 38 patients. Interestingly, these were followed by intensification of therapy in $10(26 \%)$ patients (reasons for this will be explained in detail below). Treatment was left unchanged in the majority of patients (52\%).

By performing a crosstable analysis, we could demonstrate a significant correlation between the 3 TL groups and the resulting adaptations of anti-TNF therapy (Pearson's $\left.\chi^{2}=12.03, p<0.01\right)$. Analyzing the 3 TL groups independently, significant adaptations of anti-TNF therapy were only evident in the subtherapeutic TL group (exact binomial test, 2 -sided, $p=0.01$ ), but not in the other 2 groups (Table 2). Other treatment adaptations (i.e., not involving anti-TNF therapy changes) were performed in $51 \%$ of the subtherapeutic, $33 \%$ of the therapeutic, and $24 \%$ of patients of the supratherapeutic TL groups $(p=$ $0.04)$.

When subdividing the reasons for TL measurements (Fig. 1) into the subsequent TL results, it became apparent that of the 65 patients (out of 104 in total) presenting with a clinical surrogate for increased disease activity (clinical flare, a sonographic or endoscopic activity, or elevated laboratory parameters), only 19 patients (29\%) actually had subtherapeutic TL (Table 3). Twenty-five (38\%) and $21(32 \%)$ patients had therapeutic and supratherapeutic TLs, respectively $(p=0.16)$. This analysis shows that in our patient collective, there is no evidence for a correlation between disease activity and TL results.

Trough Level and Anti-Drug Antibody Measurement in IBD

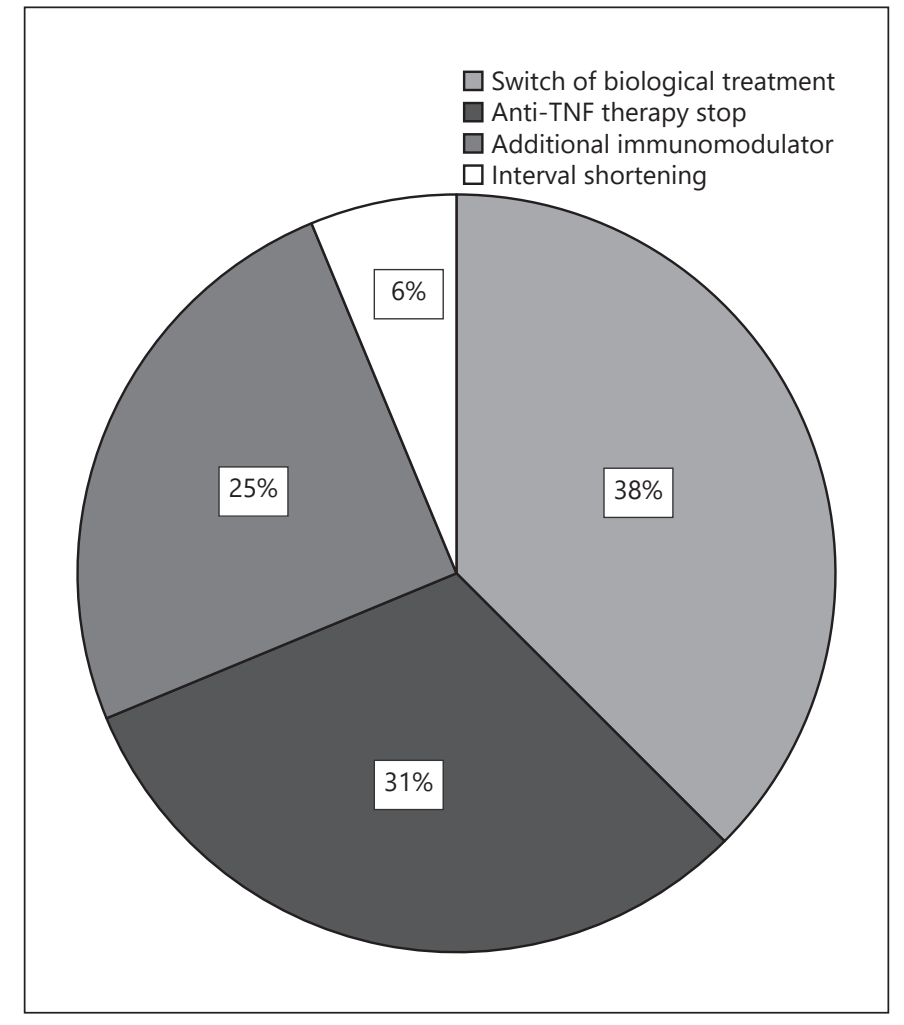

Fig. 3. Presence of ADAs and therapeutic consequences. Switch in biological treatment $(n=6)$ to infliximab, adalimumab, certolizumab pegol, or golimumab. Stopping anti-TNF therapy $(n=5$; due to quiescent disease $[n=3]$ and preparation for surgery $[n=2])$. Additional immunomodulatory treatment $(n=4)$ : AZA and MTX. Interval shortening $(n=1)$. ADAs, anti-drug antibodies; TNF, tumor necrosis factor; AZA, azathioprine; MTX, methotrexate.

\section{Therapeutic Consequences of ADA Measurements}

ADA levels were determined in all 104 patients. Presence of ADA according to TL subgroups is presented in Table 4. Sixteen patients (14\%) had positive ADA titers, and as expected, 15 of them presented with subtherapeutic TL results. Thirteen of the 64 patients treated with infliximab (20\%) and 3 of the 40 patients treated with adalimumab $(8 \%)$ developed ADA. Therapeutic consequences of ADA presence are illustrated in Figure 3. The presence of ADA motivated to a switch in treatment in the majority of patients $(n=6)$. Moreover, to suppress the occurrence of increase of $\mathrm{ADA}, 4$ patients received the addition of an immunomodulatory treatment with AZA or MTX. ADA disappeared in 2 patients treated with MTX. Another patient received MTX but was later switched to golimumab, after the cost approval from the insurance was received. The addition of AZA was not successful in 1 patient and was switched from infliximab to adalimumab. 
Table 3. Reasons for TL measurements subdivided into corresponding TL results: Surrogates for disease activity (clinical flare, sonographic or endoscopic activity, and elevated laboratory parameters) did not correlate with trough level results $(p=0.16)$

TL subtherapeutic $(n)$ therapeutic $(n)$ supratherapeutic $(n)$
Total

Reasons for TL measurement

Clinical flare

Sonographic activity

Endoscopic activity

Elevated laboratory parameters

Total

\section{0}

rs

$\begin{array}{rrrr}10 & 15 & 13 & 38 \\ 4 & 4 & 2 & 10 \\ 3 & 1 & 2 & 6 \\ 2 & 5 & 4 & 11 \\ 19 & 25 & 21 & 65\end{array}$

TL, trough level.

Table 4. Presence of ADAs and corresponding TLs

\begin{tabular}{llrcc}
\hline & \multicolumn{2}{l}{ Presence of ADAs } & Total & $p$ value \\
\cline { 2 - 2 } & no & yes & & \\
\hline TL & & & & \\
Subtherapeutic & 18 & 15 & 33 & $<0.01$ \\
Therapeutic & 33 & 0 & 33 & $<0.01$ \\
Supratherapeutic & 37 & 1 & 38 & $<0.01$ \\
\hline Total & 88 & 16 & 104 & \\
\hline
\end{tabular}

ADAs, anti-drug antibodies; TL, trough level.

Table 5. Reasons for treatment adaptations as documented by physicians' records

\begin{tabular}{|c|c|c|c|}
\hline & \multicolumn{2}{|c|}{$\begin{array}{l}\text { Treatment adaptations } \\
\text { based on measurement of } \\
\text { TLs and/or ADAs }\end{array}$} & \multirow[t]{2}{*}{$p$ value } \\
\hline & no & yes & \\
\hline \multicolumn{4}{|l|}{$T L(n=63)$} \\
\hline Subtherapeutic & 9 & 21 & 0.05 \\
\hline Therapeutic & 9 & 6 & 0.61 \\
\hline Supratherapeutic & 9 & 9 & 1.0 \\
\hline Total & 27 & 36 & 0.31 \\
\hline
\end{tabular}

No: Treatment adaptations were based on clinical, sonographic or endoscopic activity, or laboratory values. Yes: Treatment adaptations were based on results of TL or ADAs. Only patients with anti-TNF therapy and other treatment adaptations were included. Data of the 41 patients without any treatment adaptations are not shown. TL, trough level; ADAs, anti-drug antibodies; TNF, tumor necrosis factor.
ADAs and Their Association with Infusion Reactions

ADA may be related to adverse infusion reactions [2124]. Thirteen of the 104 patients presented with suspected infusion reactions, such as a sudden asthma crisis, erythema, or pruritus during or immediately after treatment with infliximab. ADAs were found in 7 patients with infusion reactions. Thus, the odds ratio for the development of an infusion reaction in the presence of ADA was 10.6 (95\% CI 2.9-38.6, risk ratio of 5.4) compared to developing an infusion reaction without the presence of ADA. One patient had a confirmed anti-TNF therapyinduced lupus-like syndrome without the presence of ADA.

ADA development in patients initially not presenting any ADA was observed in 2 patients (treated with adalimumab) during the study period. Addition of mercaptopurine or MTX could not reverse ADA, and therefore, treatment was changed to ustekinumab.

\section{Reasons for Treatment Adjustments}

Treatment adaptions were performed in 63 out of the 104 patients (Fig. 2). As expected, most treatment adaptations were performed in the group with subtherapeutic TL. However, since treatment adaptions performed by the treating physician did not frequently correlate with clinical surrogates of disease activity (see above), we analyzed the specific reasons for the executed treatment adaptations based on our patients' health records. We investigated whether treatment adaptations were carried out primarily due to TL and/or ADA results or, in contrast, were based on findings obtained from other investigations, for example, clinical symptoms, sonography, endoscopy, or laboratory results (Table 5).
Reinhold et al. 
In the subtherapeutic TL group, 15 patients had positive ADA titers. In this group, the decision for treatment adjustment was led by TL and/or ADA results in $70 \%$. In the therapeutic TL group, no patient had ADA. In this group, the decision for treatment adjustment in order to achieve endoscopic remission was guided by TL results in $40 \%$, while in $60 \%$, other reasons were more important for treatment adaptations.

In the supratherapeutic TL group, treatment was altered in $50 \%$ due to TL results, such that the infusion intervals were extended or treatment was stopped. In the remaining 50\%, increased disease activity (based on clinical symptoms, sonography, endoscopy, or laboratory results) resulted in an intensification of anti-TNF therapy.

\section{Repetition of TL Measurements: Time Interval and}

Disease Activity Scores

In our patient collective, the average time interval between start of anti-TNF therapy and first TDM measurement was 150 days (minimum: 13 days, maximum: 700 days, SD: 148 days, IQR: 120 days). In 54 out of 104 (52\%) patients, the measurement of TL and ADA was repeated after treatment adjustments. The remaining 50 patients did not have any TDM follow-up due to quiescent disease, treatment changes to medications other than antiTNF therapy, or a complete treatment stop.

During the treatment interval, clinical scores did not change. At the time of follow-up, CD patients $(n=31)$ had a mean HBI of 5.58 (IQR: 5 ) versus 5.68 (IQR: 5 ) at the initial measurement $(p=0.88)$. UC patients $(n=15)$ had a mean MTWSI of 5.27 (IQR: 7) versus 6.87 (IQR: 6) ( $p=$ 0.13 ). By the end of the observation period, $94.7 \%$ (36 out of 38 patients) of patients presenting with supratherapeutic TL remained on the same anti-TNF therapy, 93.9\% (31 out of 33 patients) in the therapeutic TL group, and 54.5\% (18 out of 33 patients) in the subtherapeutic TL group.

\section{Discussion}

Strict guidelines for the measurement of TL and ADA in IBD patients treated with anti-TNF antibodies are missing. Accordingly, TDM might be done unnecessarily or without drawing the correct conclusions. In our present study, we retrospectively analyzed the reasons for and consequences of TL and ADA measurements in clinical routine in a large tertiary Swiss IBD center under real-world conditions. As expected, subtherapeutic TL frequently led to a treatment intensification. However, there was no correlation between TL and disease activity, and even supratherapeutic TLs

Trough Level and Anti-Drug Antibody

Measurement in IBD were followed by treatment intensification - therefore being overruled by clinical presentation of the patient. Of note, we also found that presence of ADA significantly increased the likelihood for an infusion reaction following anti-TNF antibody administration.

TL might depend on individual variations in pharmacokinetics and pharmacodynamics in each patient [2527]. Therefore, it is still unknown if the measurement of $\mathrm{TL}$, rather than peak or intermediate drug levels, is representing an adequate surrogate marker for drug concentrations [16]. Additionally, TL may be influenced by sex, serum albumin concentration, and disease activity [2830]. Furthermore, non-TNF-related inflammatory pathways have to be considered with respect to the underlying cause of disease activity [31].

In our patient collective, positive ADA titers correlated with subtherapeutic TL in 15 out of 16 patients, reflecting the known drug-neutralizing effect of $\mathrm{ADA}[4,32]$. The remaining patient with positive ADA had supratherapeutic TL and could represent a phenomenon of transient ADA development without any clinical impact $[11,33]$. The observed development of acute infusion reactions in $44 \%$ of ADA-positive patients corresponds with results found in other studies $[34,35]$. Change of anti-TNF therapy in the presence of ADA - as performed in the majority of our patient collective - is supported by other results and guidelines, which, however, demonstrated that in the presence of ADA dose increase alone leads to a therapeutic response in only a minority of patients $[9,15]$. In contrast, if no ADAs are present, therapy intensification can reinstate therapeutic response $[15,16]$.

In our clinic, the decision of treatment adjustments was primarily led by TL and ADA results (rather than by other investigations) only in the group of patients with subtherapeutic TL. These results have to be interpreted with caution, as modifications in this group were associated with the presence of ADA in 15 out of 33 patients. Subtherapeutic TLs mainly result from the drug-neutralizing effects of ADA in patients treated with infliximab and adalimumab, rather than from insufficient therapy administration [4, $24,32,36]$. Future studies should address if solely the presence of ADA - without the measurement of corresponding TL - leads to significant treatment adaptations, as the results of therapeutic and supratherapeutic TLs in our patient collective had no significant consequences on treatment adaptation. In addition, a previous study demonstrated that a longer duration of subtherapeutic TL correlated with reduced $\mathrm{ADA}$ development [37].

Treatment intensification in case of supratherapeutic $\mathrm{TL}$ and ongoing disease activity in our patient collective 
was mainly performed to achieve endoscopic remission. This is in accordance with findings of other studies [38, 39]. For supratherapeutic TL, explicit guidelines for therapy adaptation are lacking. This is partially due to the fact that until now no clear association between high or supratherapeutic TL and toxicity or specific adverse effects has been described [40]. Initially, high anti-TNF therapy concentrations have been associated with arthralgia and skin lesions as a result of a "paradoxical inflammation" [41]. This association has not been confirmed in follow-up studies [42, 43]. Additionally, it was recently demonstrated that there is no association between more frequent infections and supratherapeutic infliximab TL [44]. Our results indicate that in case of a quiescent disease and supratherapeutic drug levels, treatment intervals might be prolonged, as these adaptations have a direct impact on cost-effective medicine. However, this needs to be validated in prospective studies.

A limitation of our study is its retrospective nature as well as a potential bias in the selection of patients: by omitting any exclusion criteria, we created a heterogeneous IBD patient group, especially by summarizing CD and UC patients. This is, however, also one of the strengths of our study, since we present the real-world data and not data from a highly selected clinical trial population. Notably, $\mathrm{CD}$ and UC patient groups showed no significant differences regarding age, sex, or treatment regimen (adalimumab or infliximab). Additionally, by exclusively including patients referred to an IBD specialized tertiary clinic, we mainly selected a patient collective with a severe disease course. This fact is represented by the high number of UC patients presenting with pancolitis (E3: 43\%) and a high proportion of $\mathrm{CD}$ patients exhibiting a penetrating disease course (B3: 54\%). This might, however, also mirror a certain diligence of the treating physician in severely diseased colitis patients, who especially in those patients use the possibility of TDM. Thus, proactive TDM was mostly observed in clinicians less experienced in IBD treatment.
In conclusion, our study shows that TL and ADA are primarily used to confirm treatment decisions according to clinical disease activity. This supports a reactive rather than a proactive drug monitoring strategy. Moreover, as TLs do not correlate with disease activity, it remains difficult to establish clear guidelines for drug monitoring in clinical routine. On the other hand, proactive TDM could be performed in patients in remission, where supratherapeutic TL could justify a therapy de-escalation to save treatment costs.

\section{Statement of Ethics}

All enrolled patients were participants of the Swiss IBD Cohort study, which was approved by the Ethics Committee of the Canton Zurich (No. EK-1316) and have given their informed written consent for participation in the cohort.

\section{Conflict of Interest Statement}

The authors declare no conflicts of interest.

\section{Funding Sources}

This work was supported by grants from the Stiftung Experimentelle Biomedizin to M.S., the Swiss National Science Foundation (Grant No. 314730_166381 and Grant No.320030_184753) to M.S., and the Hochspezialisierte Medizin Schwerpunkt Immunologie (HSM-2-Immunologie) to O.B.

\section{Author Contributions}

M.S. and I.R. conceived and designed the project. I.R. collected all data. M.S. supervised the project. I.R. and S.B. wrote the manuscript. I.R., J.B., and M.C. performed statistical analyses. J.S. and O.B. planned and analyzed TL and ADA measurements. I.R., S.B., and M.S. discussed the results and reviewed the manuscript. All authors approved the final version of the manuscript.

\section{References}

1 Mehta F. Report: economic implications of inflammatory bowel disease and its management. Am J Manag Care. 2016 Mar;22(3 Sup$\mathrm{pl}):$ s51-60.

2 Mitrev N, Vande Casteele N, Seow CH, Andrews JM, Connor SJ, Moore GT, et al. Review article: consensus statements on therapeutic drug monitoring of anti-tumour necrosis factor therapy in inflammatory bowel diseases. Aliment Pharmacol Ther. 2017 Dec;46(1112):1037-53.
3 Boyman O, Comte D, Spertini F. Adverse reactions to biologic agents and their medical management. Nat Rev Rheumatol. 2014 Oct; 10(10):612-27.

4 Baert F, Noman M, Vermeire S, Van Assche G, D'Haens G, Carbonez A, et al. Influence of immunogenicity on the long-term efficacy of infliximab in Crohn's disease. N Engl J Med. 2003 Feb 13;348(7):601-8.
5 Ford AC, Sandborn WJ, Khan KJ, Hanauer SB, Talley NJ, Moayyedi P. Efficacy of biological therapies in inflammatory bowel disease: systematic review and meta-analysis. Am J Gastroenterol. 2011 Apr;106(4):644-60; .

6 Sprakes MB, Ford AC, Warren L, Greer D, Hamlin J. Efficacy, tolerability, and predictors of response to infliximab therapy for Crohn's disease: a large single centre experience. J Crohns Colitis. 2012 Mar;6(2):143-53. 
7 Roda G, Jharap B, Neeraj N, Colombel JF. Loss of response to anti-TNFs: definition, epidemiology, and management. Clin Transl Gastroenterol. 2016;7(1):e135-35.

8 Harbord M, Eliakim R, Bettenworth D, Karmiris K, Katsanos K, Kopylov U, et al. Third European evidence-based consensus on diagnosis and management of ulcerative colitis. Part 2: current management. J Crohns Colitis. 2017 Jul 1;11(7):769-84.

9 Papamichael K, Cheifetz AS, Melmed GY, Irving PM, Casteele NV, Kozuch PL, et al. Appropriate therapeutic drug monitoring of biologic agents for patients with inflammatory bowel diseases. Clin Gastroenterol Hepatol. 2019 Aug;17(9):1655-68.e3.

10 Baert F, Drobne D, Gils A, Vande Casteele N, Hauenstein S, Singh S, et al. Early trough levels and antibodies to infliximab predict safety and success of reinitiation of infliximab therapy. Clin Gastroenterol Hepatol. 2014 Sep; 12(9):1474-81.e2; .

11 Ungar B, Chowers Y, Yavzori M, Picard O, Fudim E, Har-Noy O, et al. The temporal evolution of antidrug antibodies in patients with inflammatory bowel disease treated with infliximab. Gut. 2014 Aug;63(8):1258-64.

12 Vande Casteele N, Gils A, Singh S, Ohrmund L, Hauenstein S, Rutgeerts P, et al. Antibody response to infliximab and its impact on pharmacokinetics can be transient. Am J Gastroenterol. 2013 Jun;108(6):962-71.

13 Paul S, Del Tedesco E, Marotte H, RinaudoGaujous M, Moreau A, Phelip JM, et al. Therapeutic drug monitoring of infliximab and mucosal healing in inflammatory bowel disease: a prospective study. Inflamm Bowel Dis. 2013 Nov;19(12):2568-76.

14 Silva-Ferreira F, Afonso J, Pinto-Lopes P, Magro F. A systematic review on infliximab and adalimumab drug monitoring: levels, clinical outcomes and assays. Inflamm Bowel Dis. 2016 Sep;22(9):2289-301.

15 Afif W, Loftus EV Jr, Faubion WA, Kane SV, Bruining DH, Hanson KA, et al. Clinical utility of measuring infliximab and human antichimeric antibody concentrations in patients with inflammatory bowel disease. Am J Gastroenterol. 2010 May;105(5):1133-9.

16 Papamichael K, Cheifetz AS. Use of anti-TNF drug levels to optimise patient management. Frontline Gastroenterol. 2016 Oct;7(4):289300.

17 Tighe D, McNamara D. Clinical impact of immunomonitoring in the treatment of inflammatory bowel disease. World J Gastroenterol. 2017 Jan 21;23(3):414-25.

18 Truelove SC, Witts LJ. Cortisone in ulcerative colitis. Br Med J. 1955;2(4947):1041.

19 Harvey RF, Bradshaw JM. A simple index of Crohn's-disease activity. Lancet. 1980; 1(8167):514.

20 Vande Casteele N, Ferrante M, Van Assche G, Ballet V, Compernolle G, Van Steen K, et al. Trough concentrations of infliximab guide dosing for patients with inflammatory bowel disease. Gastroenterology. 2015 Jun;148(7): 1320-e3.

21 Cheifetz A, Mayer L. Monoclonal antibodies, immunogenicity, and associated infusion reactions. Mt Sinai J Med. 2005 Jul;72(4):250-6.

22 O’Meara S, Nanda KS, Moss AC. Antibodies to infliximab and risk of infusion reactions in patients with inflammatory bowel disease: a systematic review and meta-analysis. Inflamm Bowel Dis. 2014 Jan;20(1):1-6.

23 Lichtenstein L, Ron Y, Kivity S, Ben-Horin S, Israeli E, Fraser GM, et al. Infliximab-related infusion reactions: systematic review. J Crohns Colitis. 2015;9(9):806-15.

24 Gorovits B, Baltrukonis DJ, Bhattacharya I, Birchler MA, Finco D, Sikkema D, et al. Immunoassay methods used in clinical studies for the detection of anti-drug antibodies to adalimumab and infliximab. Clin Exp Immunol. 2018;192(3):348-65.

25 Wang W, Wang EQ, Balthasar JP. Monoclonal antibody pharmacokinetics and pharmacodynamics. Clin Pharmacol Ther. 2008 Nov; 84(5):548-58.

26 Fasanmade AA, Adedokun OJ, Ford J, Hernandez D, Johanns J, Hu C, et al. Population pharmacokinetic analysis of infliximab in patients with ulcerative colitis. Eur J Clin Pharmacol. 2009 Dec;65(12):1211-28.

27 Vande Casteele N, Gils A. Pharmacokinetics of anti-TNF monoclonal antibodies in inflammatory bowel disease: adding value to current practice. J Clin Pharmacol. 2015 Mar; 55(Suppl 3):S39-50.

28 Ben-Horin S, Chowers Y. Tailoring anti-TNF therapy in IBD: drug levels and disease activity. Nat Rev Gastroenterol Hepatol. 2014 Apr; 11(4):243-55.

29 Dotan I, Ron Y, Yanai H, Becker S, Fishman S, Yahav L, et al. Patient factors that increase infliximab clearance and shorten half-life in inflammatory bowel disease: a population pharmacokinetic study. Inflamm Bowel Dis. 2014 Dec;20(12):2247-59.

30 Buurman DJ, Maurer JM, Keizer RJ, Kosterink JG, Dijkstra G. Population pharmacokinetics of infliximab in patients with inflammatory bowel disease: potential implications for dosing in clinical practice. Aliment Pharmacol Ther. 2015 Sep;42(5):529-39.

31 Allez M, Karmiris K, Louis E, Van Assche G, Ben-Horin S, Klein A, et al. Report of the ECCO pathogenesis workshop on anti-TNF therapy failures in inflammatory bowel diseases: definitions, frequency and pharmacological aspects. J Crohns Colitis. 2010 Oct; 4(4):355-66.

32 Bartelds GM, Krieckaert CL, Nurmohamed MT, van Schouwenburg PA, Lems WF, Twisk JW, et al. Development of antidrug antibodies against adalimumab and association with disease activity and treatment failure during long-term follow-up. JAMA. 2011 Apr 13; 305(14):1460-8.

33 Steenholdt C, Al-khalaf M, Brynskov J, Bendtzen K, Thomsen OØ, Ainsworth MA. Clinical implications of variations in anti-infliximab antibody levels in patients with inflammatory bowel disease. Inflamm Bowel Dis. 2012 Dec; 18(12):2209-17.

34 Maneiro JR, Salgado E, Gomez-Reino JJ. Immunogenicity of monoclonal antibodies against tumor necrosis factor used in chronic immune-mediated inflammatory conditions: systematic review and meta-analysis. JAMA Intern Med. 2013 Aug 12;173(15):1416-28.

35 Warman A, Straathof JW, Derijks LJ. Therapeutic drug monitoring of infliximab in inflammatory bowel disease patients in a teaching hospital setting: results of a prospective cohort study. Eur J Gastroenterol Hepatol. 2015 Mar;27(3):242-8.

36 Hanauer SB, Wagner CL, Bala M, Mayer L, Travers S, Diamond RH, et al. Incidence and importance of antibody responses to infliximab after maintenance or episodic treatment in Crohn's disease. Clin Gastroenterol Hepatol. 2004 Jul;2(7):542-53.

37 Brandse JF, Mould D, Smeekes O, Ashruf Y, Kuin S, Strik A, et al. A real-life population pharmacokinetic study reveals factors associated with clearance and immunogenicity of infliximab in inflammatory bowel disease. Inflamm Bowel Dis. 2017 Apr;23(4):650-60.

38 Imaeda H, Bamba S, Takahashi K, Fujimoto T, Ban H, Tsujikawa T, et al. Relationship between serum infliximab trough levels and endoscopic activities in patients with Crohn's disease under scheduled maintenance treatment. J Gastroenterol. 2014 Apr;49(4):674-82.

39 Barnes EL, Allegretti JR. Are anti-tumor necrosis factor trough levels predictive of mucosal healing in patients with inflammatory bowel disease?: a systematic review and meta-analysis. J Clin Gastroenterol. 2016 Oct;50(9):733-41.

40 McLean LP, Cross RK. Adverse events in IBD: to stop or continue immune suppressant and biologic treatment. Expert Rev Gastroenterol Hepatol. 2014;8(3):223-40.

41 Cleynen I, Vermeire S. Paradoxical inflammation induced by anti-TNF agents in patients with IBD. Nat Rev Gastroenterol Hepatol. 2012 Sep;9(9):496-503.

42 Brandse JF, Vos LM, Jansen J, Schakel T, Ponsioen CI, van den Brink GR, et al. Serum concentration of anti-TNF antibodies, adverse effects and quality of life in patients with inflammatory bowel disease in remission on maintenance treatment. J Crohns Colitis. 2015;9(11):973-81.

43 Coutzac C, Chapuis J, Poullenot F, Chabrun E, Capdepont M, Blanco P, et al. Association between infliximab trough levels and the occurrence of paradoxical manifestations in patients with inflammatory bowel disease: a case-control study. J Crohns Colitis. 2015 Nov;9(11):982-7.

44 Greener T, Kabakchiev B, Steinhart AH, Silverberg MS. Higher infliximab levels are not associated with an increase in adverse events in inflammatory bowel disease. Inflamm Bowel Dis. 2018 Jul 12;24(8):1808-14. 